\title{
Investigation of facial motor pathways by electrical and magnetic stimulation: sites and mechanisms of excitation
}

\author{
K M RÖSLER, C W HESS, U D SCHMID* \\ From the Departments of Neurology and Neurosurgery, * University of Bern, Inselspital, CH-3010 Bern, \\ Switzerland
}

SUMMARY A refined technique is described for non invasive examination of the facial motor pathways by stimulation of the extra- and intracranial segment of the facial nerve and the facial motor cortex. Surface recordings from the nasalis muscle rather than from the orbicularis oris muscle were used, since the compound muscle action potential (CMAP) from this muscle showed a more clearly defined onset. Electrical extracranial stimulation of the facial nerve at the stylomastoid fossa in 14 healthy subjects yielded a mean distal motor latency of $3.7 \mathrm{~ms}$ (SD 0.46), comparable with reported latencies to the orbicularis oris muscle. Using a magnetic stimulator, transcranial stimulation of the facial nerve was performed. The mechanism of transcranial magnetic facial nerve stimulation was studied using recordings on 12 patients who had facial nerve lesions at different locations, and with intraoperative direct measurements in four patients undergoing posterior fossa surgery. The actual site of stimulation could be localised to the proximal part of the facial canal, and a mean "transosseal conduction time" of $1.2 \mathrm{~ms}$ (SD $0 \cdot 18$ ) was calculated. The cerebrospinal fluid (CSF) played an important role in mediating the magnetically induced stimulating currents. Finally, with transcranial magnetic stimulation of the facial motor cortex, clearly discernible CMAPs could be produced when voluntary activation of several facial muscles was used to facilitate the responses. From this, a central motor conduction time of $5 \cdot 1 \mathrm{~ms}$ was calculated (SD 0.60, 6 subjects).

Motor neurography is routinely used to assess facial nerve function,' and is considered an important means of estimating the outcome in facial nerve palsies of various aetiologies. ${ }^{2}$ Until recently, only the extracranial part of the nerve was accessible to neurography. Many facial nerve palsies are, however, caused by lesions located in the intracranial segment of the nerve. Since the first report by Murray et $a l^{3}$ in 1987 on magnetic facial nerve stimulation it is possible to stimulate this cranial nerve, non invasively, within the cranium. ${ }^{4}$ Furthermore, the same stimulation technique has been used to excite the human motor

Correspondence to: Dr C W Hess, Neurologische Klinik, Inselspital, CH-3010 Bern, Switzerland.

Received 28 October 1988 and in revised form 25 March 1989. Accepted 10 May 1989 cortex transcranially, ${ }^{3}$ and it has been shown that the central pathways supplying the facial muscles can also be stimulated. ${ }^{6}$ The precise mechanisms and actual sites of transcranial facial nerve stimulation are, however, still controversial, ${ }^{7}$ and the optimal stimulating technique and recording parameters for standardised procedures have not been formulated. This study aims to clarify the mechanisms of transcranial facial nerve stimulation and to assess the clinical application of the method.

\section{Method}

The experiments complied with the standards of the local ethical committee. Fifteen healthy subjects gave their informed consent and volunteered for the experiments (eight males and seven females, mean age 29, range 24 to 42 years). In addition, eight patients with Bell's palsy and four patients with facial palsies of other aetiologies were studied at various intervals after the onset of the palsy. Intraoperative 
measurements were carried out in another four patients receiving posterior fossa surgery.

In a preliminary series of seven subjects, surface electromyograms (SEMG) from both the nasalis (the recording procedure is described later) and the orbicularis oris muscles were simultaneously recorded, the latter being the most commonly used muscle for facial nerve neurography. ${ }^{124}$

For the other experiments, recordings from the nasalis muscle with two different recording procedures were used. The first was a one channel recording from two surface electrodes taped symmetrically over both nasalis muscles, where the electrode on the contralateral muscle served as a reference. This procedure was used only for experiments with peripheral facial nerve stimulation and gave a side to side comparison similar to the method described for the orbicularis oris muscle. ${ }^{2}$ The second procedure was a two channel recording used for experiments where additional cortical stimulation was performed. A common reference electrode was taped to the tip of the nose. This gave a separate surface recording of the two nasalis muscles on each side, without interference from a possibly coactivated contralateral muscle. Conduction latencies were measured from the stimulus to the negative onset of the CMAP.

For extracranial electrical stimulation of the facial nerve, the cathode was placed at the stylomastoid fossa. ${ }^{14} \mathrm{~A}$ magnetic stimulator was used for transcranial stimulation of either the nerve ${ }^{34}$ or the motor cortex. ${ }^{8}$ It had a total capacitance of $800 \mu \mathrm{F}$ and used a total charging energy of 2500 Joules with $2.5 \mathrm{kV}$ at maximum output. The stimulating coil had a mean diameter of $8.1 \mathrm{~cm}$. For transcranial peripheral nerve stimulation, the magnetic coil was placed tangentially on the parieto-occipital surface of the scalp. The stimulus intensity was then increased stepwise until a supramaximal response was obtained.

To determine the site of the intracranial excitation more precisely, experiments were carried out on four patients before and during posterior fossa surgery. In cases one and two, measurements could be taken on an anatomically intact facial nerve, while in cases three and four, the nerve was found to be slightly compressed by an acoustic neurinoma. During surgery, surface recordings were made from the nasalis muscles and a needle electrode was inserted close to the stylomastoid segment of the facial nerve.' This needle electrode was used for both the extracranial stimulation of the nerve and recording the compound nerve action potential (CNAP) after direct stimulation of the intracisternal segment of the nerve. After microsurgical exposure, the intracisternal segment of the facial nerve was stimulated directly using a bipolar stimulating forceps which was insulated except for the tips. ${ }^{9}$ For comparison, magnetic stimulation of the facial nerve was also repeatedly performed during the operation.

Stimulation of the motor cortex was carried out as described earlier. ${ }^{81011}$ The centre of the stimulating coil was placed $2-3 \mathrm{~cm}$ paramedially to the vertex over the stimulated hemisphere. A variety of manoeuvres were tested for their facilitatory effect on the target muscle response. These included background contraction of the target (nasalis) muscle alone or simultaneously with contractions of the tongue (pushing the tongue to the palate, sticking the tongue out) or the masseter muscles (biting the teeth). In order to minimise the baseline irregularities from continuing activity and render latency measurements more reliable, two to eight responses were averaged.
Results

\section{Preliminary experiments}

In the orbicularis oris muscle, a clear negative onset of the CMAP from a horizontal baseline was found in only three of the 14 sides. Despite repeated attempts to improve the electrode position, an initial positive deflection rendered measurement of the CMAP onset difficult in the remaining 11 sides (figure 1). In contrast, recordings from the nasalis muscle yielded a clearly definable negative onset in all 14 sides. The relative side to side differences in the amplitudes were similar and ranged from $0 \%$ to $31 \%$ for the orbicularis oris, and from $0 \%$ to $37 \%$ for the nasalis muscle.

\section{Peripheral facial nerve stimulation}

Precise positioning of the electrical stimulation electrode at the stylomastoid fossa proved to be crucial for

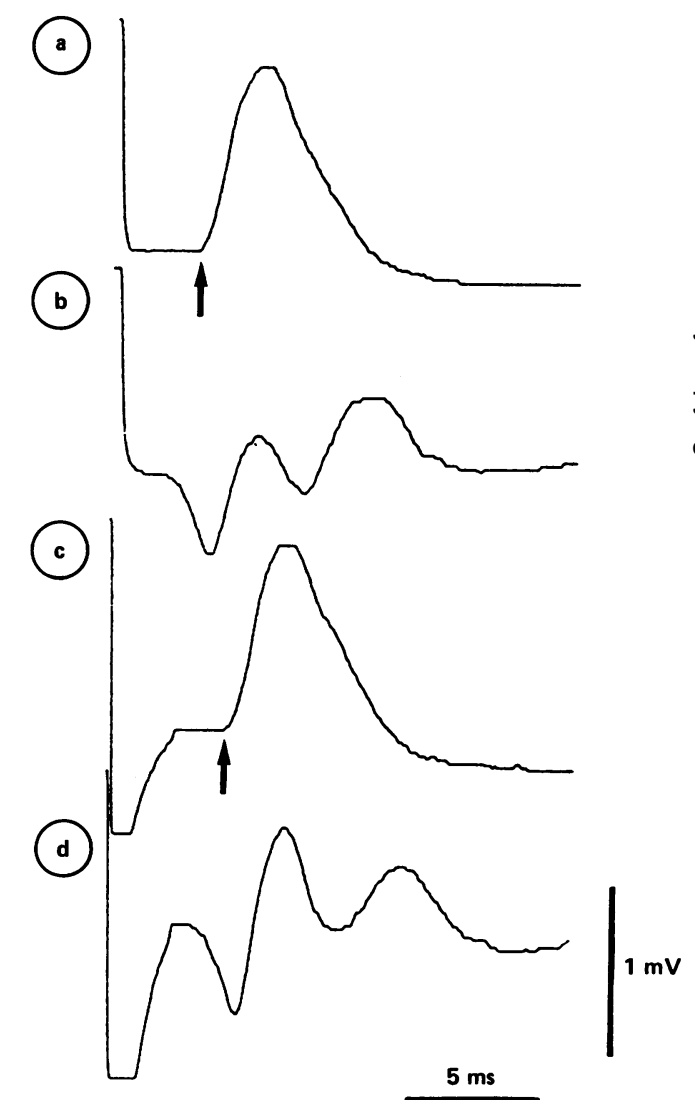

Fig 1 Recordings from the nasalis $(a, c)$ and orbicularis oris muscle $(b, d)$ of a healthy subject after electrical stimulation of the facial nerve at the stylomastoid fossa $(a, b)$ and transcranial magnetic stimulation $(c, d)$. Latencies (arrowed) were $3.82 \mathrm{~ms}(a)$ and $5.82 \mathrm{~ms}(\mathrm{c})$. 
Table 1 Results from peripheral facial nerve stimulation. Onset latencies and amplitudes from the nasalis muscle following electrical stylomastoid and magnetic transcranial stimulation. Mean values (SD) are given. Our results are compared with the data of Schriefer, et al ${ }^{4}$

\begin{tabular}{|c|c|c|c|c|c|}
\hline & $\begin{array}{l}\text { Right side } \\
(n=14)\end{array}$ & $\begin{array}{l}\text { Left side } \\
(n=14)\end{array}$ & $\begin{array}{l}\text { Right/left- } \\
\text { difference }\end{array}$ & $\begin{array}{l}\text { Both sides } \\
(n=28)\end{array}$ & $\begin{array}{l}\text { Schriefer et al } \\
(n=30)\end{array}$ \\
\hline $\begin{array}{l}\text { Stylomastoid } \\
\text { Transcranial } \\
\text { Transosseal conduction time }\end{array}$ & $\begin{array}{ll}3 \cdot 6 & (0 \cdot 36) \\
4 \cdot 8 & (0.45) \\
1 \cdot 2 & (0 \cdot 16)\end{array}$ & $\begin{array}{ll}3.8 & (0 \cdot 53) \\
4.9 & (0 \cdot 53) \\
1 \cdot 2 & (0 \cdot 18)\end{array}$ & $\begin{array}{ll}0.3 & (0 \cdot 36)^{*} \\
0.3 & (0 \cdot 27)^{*} \\
0.2 & (0 \cdot 20)^{*}\end{array}$ & $\begin{array}{ll}3.7 & (0.46) \\
4.9 & (0.50) \\
1.2 & (0.18)\end{array}$ & $\begin{array}{ll}3 \cdot 8 & (0 \cdot 75) \\
5.1 & (0 \cdot 76) \\
1.3 & (0 \cdot 15)\end{array}$ \\
\hline $\begin{array}{l}\text { Amplitude (mV): } \\
\text { Stylomastoid } \\
\text { Transcranial } \\
\text { Transosseal difference }\end{array}$ & $\begin{array}{ll}3.3 & (0.99) \\
3.3 & (1.02) \\
0.1 & (0.27)\end{array}$ & $\begin{array}{ll}3 \cdot 3 & (1 \cdot 20) \\
3 \cdot 2 & (1 \cdot 02) \\
0 \cdot 1 & (0 \cdot 28)\end{array}$ & $\begin{array}{ll}0.6 & (0.44)^{*} \\
0.5 & (0.53)^{*} \\
0.3 & (0.25)^{*}\end{array}$ & $\begin{array}{ll}3 \cdot 3 & (1 \cdot 10) \\
3 \cdot 2 & (1 \cdot 02) \\
0 \cdot 1 & (0 \cdot 28)\end{array}$ & $\begin{array}{r}0.5-2 \cdot 8 \\
0.4-2.2 \\
-0.4-0.7\end{array}$ \\
\hline
\end{tabular}

${ }^{*} \mathrm{p}>0.05$, paired $t$ test.

reliable latency measurements. In all the subjects a very localised pressure sensitive point directly behind the mandibular angle was optimal for electrical stimulation. Stimulation a few millimeters away from this point required considerably higher stimulus intensities and resulted in a variably pronounced, unpredictable and sometimes dramatic shortening of the latency of the CMAP.

For magnetic transcranial stimulation of the facial nerve, the orientation of the inducing current rather than the positioning of the stimulating coil was critical. The current in the coil was anticlockwise (when viewed from outside) to stimulate the right facial nerve and vice versa. By doing this, it proved easy to stimulate the facial nerve supramaximally, and CMAPs of similar shape and amplitude from stylomastoidal stimulation were obtained. The latencies were on the average longer by $1.2 \mathrm{~ms}$ (table 1). They were constant for a given subject and did not depend on the precise position of the stimulating coil, provided the coil was not moved too close to the retromandibular region. In the latter case, the response moved to a shorter latency which was virtually identical to the latency obtained from stylomastoidal stimulation. This indicates that magnetic transcranial stimulation excited the nerve at an individually constant site, and made it easy to find an appropriate coil position.
In all eight patients with acute Bell's palsy, a muscular response in the nasalis muscle could be evoked by stylomastoid electrical stimulation, but not with transcranial magnetic stimulation of the facial nerve (table 2). Repeated examinations during follow up showed a persisting lack of response of the facial nerve to transcranial magnetic stimulation even after an appreciable recovery from the palsy to near normal or complete functioning in six of the eight patients; in the two remaining patients only a small response could be evoked (table 2).

In a patient with a complete traumatic facial nerve palsy due to a pyramidal fracture, a normal response of $2.5 \mathrm{mV}$ amplitude and $5.0 \mathrm{~ms}$ latency was obtained from stylomastoid electrical stimulation, but none from magnetic stimulation one day after the trauma. Five days later the response from stylomastoid stimulation had diminished to an amplitude of $0.4 \mathrm{mV}$. In two patients with partial nerve lesions after surgical removal of an acoustic neurinoma, there was a diminished CMAP amplitude five or six days after surgery with both stylomastoid and magnetic nerve stimulation (fig 2). Finally, in a patient with a transient facial palsy due to a demyelinating brainstem lesion, normal responses were elicited with stylomastoid and magnetic nerve stimulation.

In four patients, transcranial magnetic and direct electrical facial nerve stimulation were performed

able 2 Facial nerve conduction studies from eight patients with Bell's palsy: (mean values (SD))

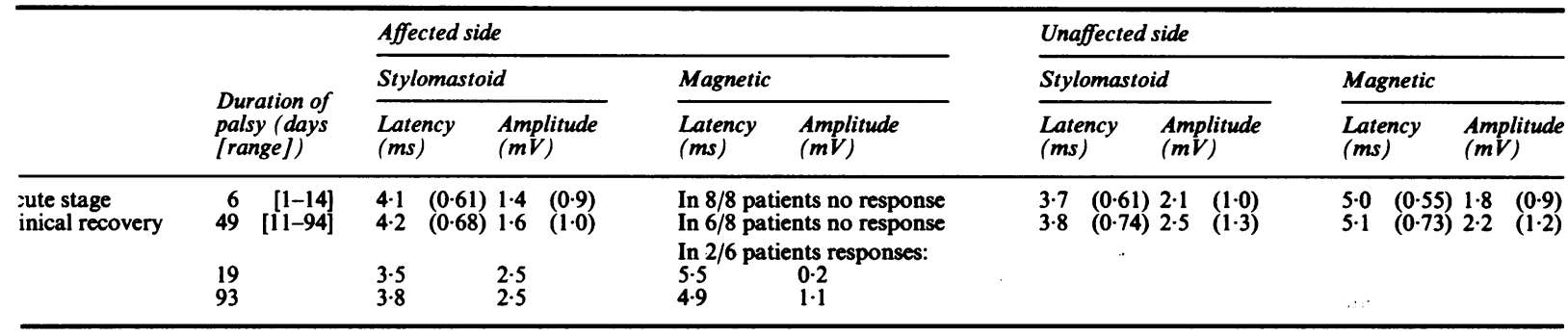



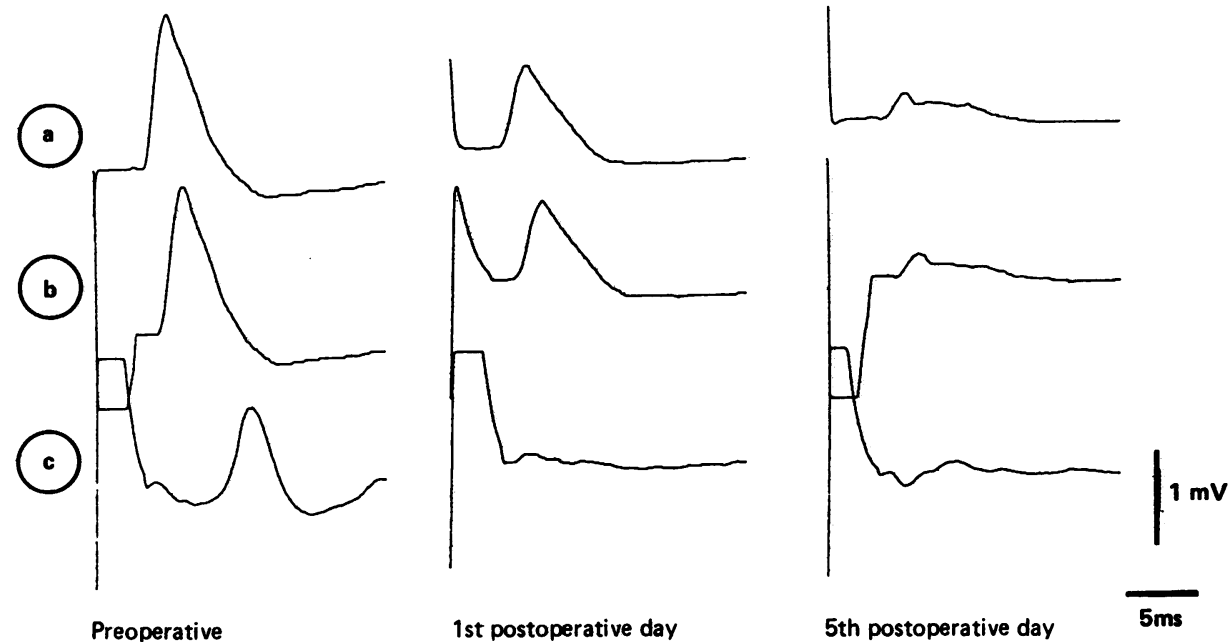

Fig 2 Serial examinations of a patient before and one and five days after removal of an acoustic neurinoma. Recordings after electrical stimulation at the stylomastoid fossa $(a)$ and transcranial magnetic stimulation of the peripheral nerve $(b)$ and of the facial motor cortex $(c)$. Immediately after surgery a clinically almost complete facial palsy was present which began to recover slightly after five days.

during posterior fossa surgery. Direct electrical stimulation of the nerve in its intracisternal segment at its entrance into the facial canal yielded latencies and transosseal conduction times which were equal or slightly longer compared with transcranial magnetic stimulation (table 3, figs 3 and 4). In two cases, we removed the cerebrospinal fluid. In these cases the nerve proved clearly less excitable with the stimulating coil placed at the usual position over the scalp: in case number one a considerably higher stimulus intensity $(+80 \%)$ was needed to get a supramaximal response, and in case number two no response could be elicited after removing the CSF. This finding could be reproduced by refilling the cisterna cerebellopontina with Ringer solution and by taking it out again.

\section{Motor cortex stimulation}

As for exciting small hand muscles, transcrania

Table 3 Results from pre-and intraoperative facial nerve stimulation in four patients with posterior fossa surgery. Onset latencies (mas) the compound muscle action potential (MAP) from the nasalis muscle and simultaneously recorded facial compound nerve action potentian (NAP) from the stylomastoid segment of the nerve. Calculated conduction times (CT) between intracranial and extracranial nerve stimulation (nd $=$ not done)

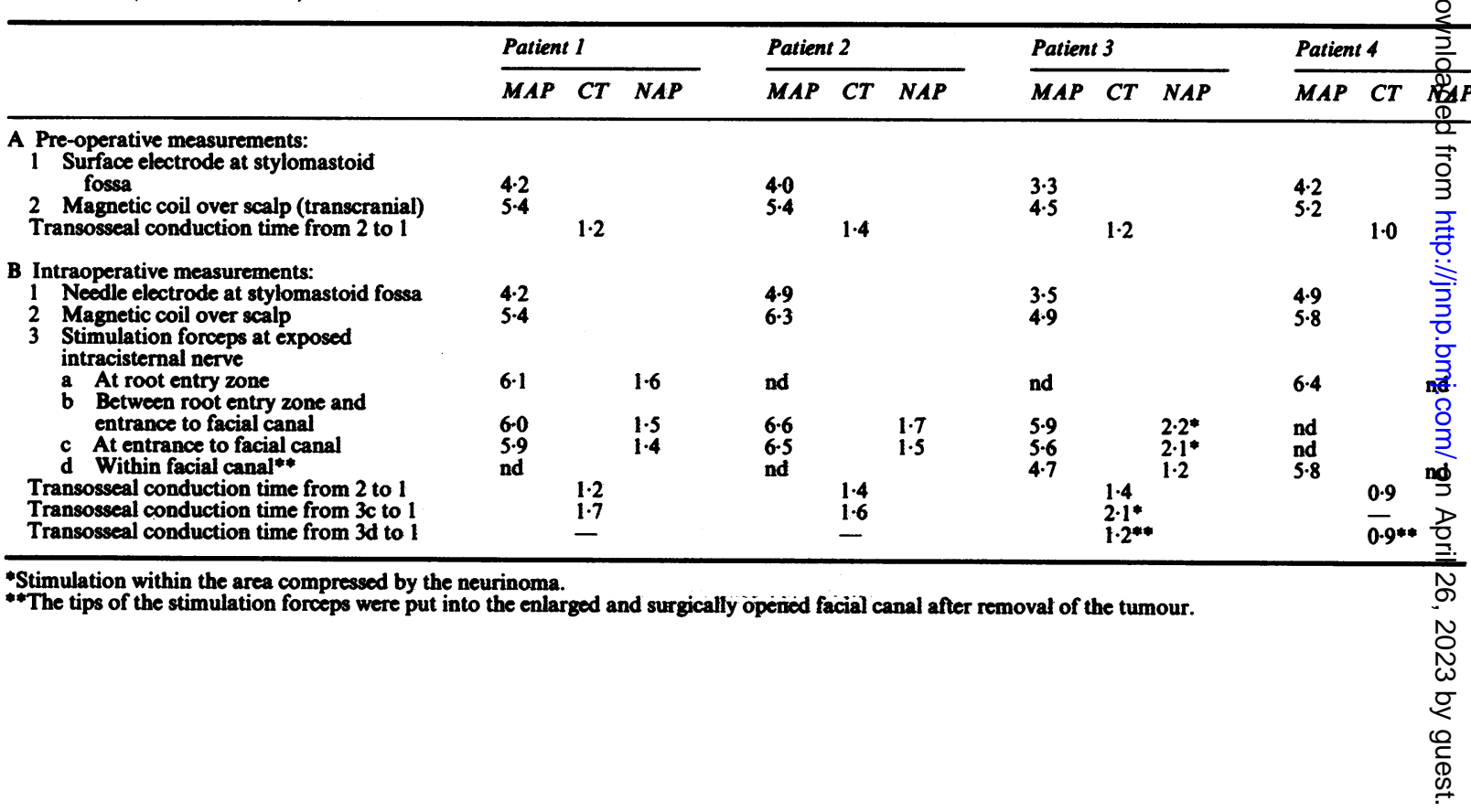




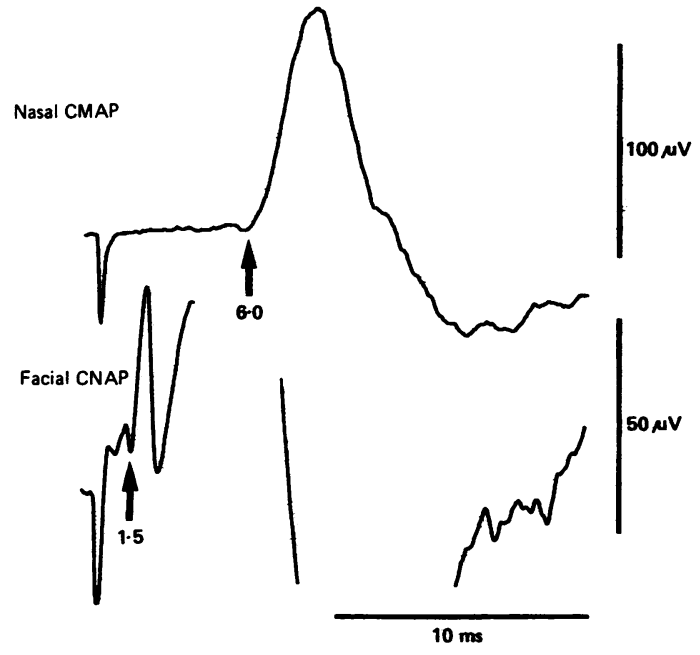

Fig 3 Intraoperative measurements of case one (see text and table 3). Simultaneous recording of compound muscle action potential (CMAP) from the nasalis muscle and compound nerve action potential (CNAP) from the facial nerve at the stylomastoid fossa following direct intracisternal nerve stimulation.

magnetic cortex stimulation of the nasalis muscle required a clockwise current flow in the coil as viewed from above to excite the right-sided muscle and vice versa. A stimulus intensity just below the threshold for transcranial magnetic stimulation of the facial nerve on the side aimed at had to be chosen to avoid superimposition of a peripheral response. With this intensity, no response could be evoked by cortical stimulation in a relaxed nasalis muscle. Since facilitation by voluntary activation is a known characteristic of responses from motor cortex stimulation, ${ }^{1213}$ a voluntary background contraction was exerted during cortex stimulation. In most subjects background contraction of the target (nasalis) muscle alone was not sufficient and additional contraction of either the tongue or the masticatory muscles or both were needed to obtain a response. As for responses from upper limb muscles, the shape and amplitude of these cortically evoked CMAPs were not comparable with the CMAPs from either magnetic or electrical peripheral nerve stimulation. ${ }^{81014}$ From the cortex to muscle latency and from the latency of transcranial magnetic facial nerve stimulation, a central conduction time was calculated (figures 2,5 ; table 4 ). In three patients who had clinically recovered from their Bell's palsy, but whose facial nerves were still unresponsive to transcranial magnetic stimulation, centrally evoked responses were readily obtained by cortical stimulation (figure 5).

\section{Discussion}

Stylomastoid stimulation of the facial nerve with recordings from the orbicularis oris muscle is a widely used method to assess facial palsies. ${ }^{2}$ We describe a refined magnetic stimulation technique which enables additional stimulation of the facial motor pathways at two different sites within the cranium.

For the surface recordings, the nasalis muscle proved clearly superior to the orbicularis oris muscle since a clear cut negative onset of the CMAPs allowed a more reliable latency measurement. Recordings from the nasalis muscle seemed less prone to pick up volume conducted activity from neighbouring muscles, probably because of their more isolated topography. This is particularly important for magnetic stimulation of the facial nerve, as other cranial nerves are also stimulated by this technique. ${ }^{6}$ Recordings from the nasalis muscle have a further advantage of being at a greater distance from the stimulating electrodes and stimulating coil, thus keeping the stimulus artifact smaller. The latencies and amplitudes obtained from electrical stylomastoid stimulation and the surface recordings from the nasalis muscle were similar to those reported for the orbicularis oris muscle. 2415

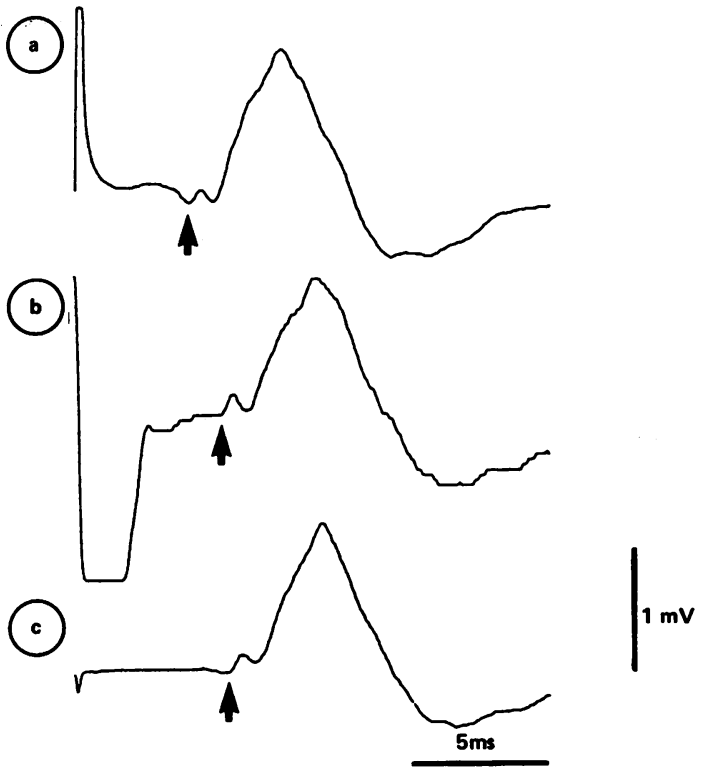

Fig 4 Intraoperative measurement of case two (see text and table 3). Recordings of compound muscle action potentials (CMAP) from the nasalis muscle following electrical stimulation at the stylomastoid fossa (a) transcranial magnetic stimulation (b) and direct electrical stimulation at. .. the entrance of the nerve into the facial canal (c). Onset latencies (arrowed) were $4.88 \mathrm{~ms}(\mathrm{a}), 6.24 \mathrm{~ms}(b)$, and $6.48 \mathrm{~ms}(\mathrm{c})$. 
Table 4 Onset latencies and amplitudes from the nasalis muscle following peripheral (electric stylomastoid and magnetic transcranial) facial nerve stimulation and following stimulation of the facial motor cortex in six healthy subjects $(n=12$ sides). Calculated transosseal conduction time and central motor conduction time. Mean values (SD) are given

\begin{tabular}{|c|c|c|c|}
\hline & Stylomastoidal & Transcranial & Cortical \\
\hline $\begin{array}{l}\text { Latency (ms) } \\
\text { Amplitude (mV) }\end{array}$ & $\begin{array}{ll}3.7 & (0.35) \\
3.1 & (0.99)\end{array}$ & $\begin{array}{ll}4.9 & (0.44) \\
2.9 & (0.88)\end{array}$ & $\begin{array}{rr}10.0 & (0.96) \\
1.4 & (0.39)\end{array}$ \\
\hline \multirow{2}{*}{$\begin{array}{l}\text { Conduction time (ms) } \\
\text { Amplitude difference } \\
(\mathrm{mV})\end{array}$} & $\begin{array}{l}\text { Transosseal } \\
1.2(0 \cdot 15)\end{array}$ & $\begin{array}{l}\text { Central } \\
5 \cdot 1 \quad(0 \cdot 60)\end{array}$ & \\
\hline & $0.8 \quad(0.17)$ & $1.5 \quad(0.62)$ & \\
\hline
\end{tabular}

Using the magnetic coil, transcranial stimulation of the facial nerve was easily performed as described by Schriefer et $a l^{4}$ With the coil in an appropriate position the facial nerve could be stimulated supramaximally at a constant site within the cranium. Our calculated mean transosseal conduction time between magnetic transcranial and electrical stylomastoid stimulation of $1.2 \mathrm{~ms}$ is comparable with the $1.3 \mathrm{~ms}$ reported by Schriefer et al, ${ }^{4}$ who used recordings from the orbicularis oris muscle.

The absence of a response to magnetic stimulation in acute Bell's palsy, ${ }^{4}$ indicates an intracranial stimulation site on the nerve. From anatomical measurements and latency extrapolations, Maccabee $e t$ al, ${ }^{7}$ suggested a stimulation site close to the brain stem. However, the intriguing findings of Schriefer $\mathrm{et} \mathrm{al}^{4}{ }^{4}$ in two patients with Bell's palsy who had a complete absence of a response to magnetic nerve stimulation, even after clinical function returned to near normal, (and now confirmed in six patients of ours) has led to the conclusion that excitation occurs within the initial segment of the facial canal where the lesion is known to reside in Bell's palsy.' A still demyelinated and hence hypoexcitable target area would then explain the persisting absence of responses to magnetic stimulation.

Our four patients with acute facial palsies of different aetiology demonstrate how magnetic stimulation acts within the intratemporal rather than intracisternal segment of the nerve. In two patients with partial facial palsies after posterior fossa surgery, the recordings from the magnetic stimulation gave almost normal results one day after surgery. A few days later, a much reduced response was obtained in the same patients indicating distal degeneration of the nerve fibres typical of axonal lesions (figure 2). Since the intracisternal segment of the nerve was assumed to have been damaged by surgery, the magnetic stimulation must have acted distally to this segment. Likewise, in a patient with a transient complete facial palsy due to a demyelinating brainstem lesion, normal responses

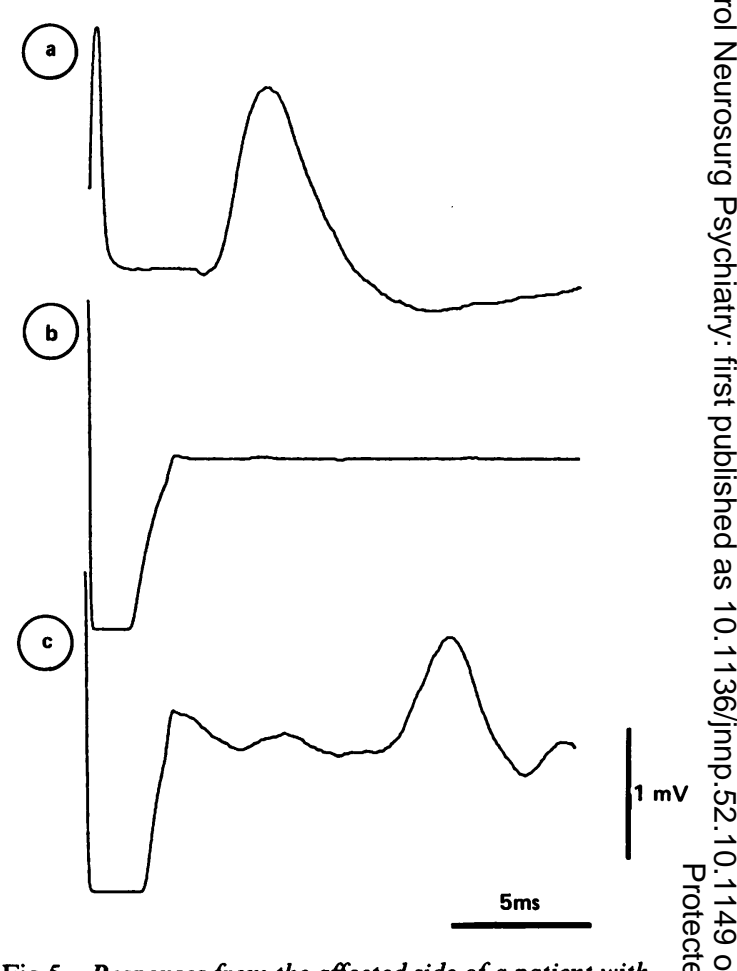

Fig 5 Responses from the affected side of a patient with Bell's palsy, 73 days after onset of the symptoms, after near complete clinical recovery. Recordings following electrical stimulation at the stylomastoid fossa (a) and transcranial magnetic stimulation of the peripheral facial nerve $(b)$ and the facial motor cortex (c).

to magnetic stimulation were repeatedly found, (as would be expected in a neurapraxic block proximal to the site of excitation). Finally, in a patient with a complete facial palsy due to a pyramidal fracture, no response was obtained from magnetic stimulation just after the accident, thus confirming that magnetic $\stackrel{\varrho}{\varrho}$ stimulation took place at or proximal to the site of the $\stackrel{\varnothing}{\triangle}$ lesion.

Also the latencies obtained from direct stimulation $\frac{0}{3}$ of the exposed intracisternal nerve and magnetic $\bar{P}$ stimulation in the same patients (table 3) during our intraoperative studies indicate that excitation occurs distal to the beginning of the facial canal, in both the affected and the unaffected facial nerves. When attempting to locate the site of excitation more 3 precisely, a rough estimate can be ventured from the $\dot{\delta}$ calculated conduction velocity of the facial nerve. The intratemporal portion of the facial nerve has a length 0 of about $30 \mathrm{~mm}^{116}$ and an additional segment of 10 to $21 \mathrm{~mm}$ from the exit from the stylomastoid fossa to the $\frac{D}{0}$ extracranial stimulation point of the nerve has been measured.9 Hence, for a total distance of $40-51 \mathrm{~mm} N$ the conduction time at operation was assessed as $N$ 
$1.6 \mathrm{~ms}$ and $1.7 \mathrm{~ms}$ (from patient one and two respectively who did not have compressed nerves, table 3 ) which gave a conduction velocity of 24 to $32 \mathrm{~m} / \mathrm{s}$. Bearing in mind that measurements at operation gave lower values, this velocity is compatible with the estimated normal facial nerve conduction velocity of 35 to $40 \mathrm{~m} / \mathrm{s}$. ' With the latency difference of 0.2 to $0.5 \mathrm{~ms}$ between direct electrical stimulation at the entrance to the facial canal and magnetic stimulation, excitation from the latter can be estimated to occur between 5 to $16 \mathrm{~mm}$ distal to the facial canal's entrance. A more direct clue is given by the studies carried out during the operations on the third and fourth patients where the tips of the stimulation forceps could be placed deeply into an enlarged facial canal to stimulate the exposed nerve within its intratemporal segment. The latencies recorded were the same or shorter by $0.2 \mathrm{~ms}$ than with magnetic stimulation, indicating that the latter does indeed excite the facial nerve in its proximal intracanalicular segment. On this evidence we conclude that magnetic stimulation excites the facial nerve somewhere within the first half of its intratemporal course, thus confirming the assumptions of Schriefer et $\mathrm{al}^{4}{ }^{4}$ and Schmid et $a l,{ }^{9}$ but contrasting with the assumption of Maccabee et al. ${ }^{7}$

Our intraoperative experiments demonstrate the important role of the (CSF) for transcranial magnetic facial nerve stimulation. Magnetic stimulation works through high resistance structures such as the skull and acts by inducing electrical currents within the tissues which bear the excited nerve fibres. It can be inferred that the magnetic pulse most easily induces currents in the CSF which is a good electrical conductor and is situated closer to the stimulating coil than the grey matter with its four to six times greater resistance. ${ }^{17}$ Eddy currents in the CSF, flowing outside the effective magnetic field, are therefore bound to occur and it is conceivable that the CSF is mediating the induced stimulating currents which excite the facial nerve. This would explain why precise coil positioning was not important for magnetic facial nerve stimulation and why much lower stimulus intensities were needed compared with the motor cortex stimulation. Since the CSF surrounds about $10 \mathrm{~mm}$ deep into the entrance of the facial canal, ${ }^{18}$ the most distal excited nerve segment could be expected to be in this region. It is possible that the stimulating currents are additionally focused by the bony hole of the facial canal. Such a focusing effect by a gap in the high resistance skull has been predicted for intracranial stimulation techniques. ${ }^{19}$

When the stimulating coil is placed near the vertex in an attempt to stimulate the facial area of the motor cortex, a peripherally evoked response will be obtained from both sides with a stimulus intensity appropriate for exciting upper limb muscles by cortex stimulation. The peripherally evoked CMAP with its long duration typical of the facial muscles (figures 1, 2-5) makes it difficult to recognise the possibility of a subsequent cortically evoked response. To get around this problem, Benecke et al, ${ }^{6}$ used concentric needle electrodes to record facial muscle activity induced by cortical stimulation. Because of the variable latencies of the dispersed activity, needle recordings are, however, not suitable for assessing conduction times and surface recordings are obviously desirable for this purpose.

With the method described here, it proved possible to show cortically evoked CMAPs recorded from the nasalis muscle by surface electrodes. Stimulus intensity, coil placement, and sense of inducing current were chosen to prevent a peripherally evoked response on the chosen side, and facilitation by voluntary contraction $^{8}$ was used in order to reveal an otherwise undetectable response. It is in fact the presence of such a facilitatory effect which, among other properties, allows the identification of the responses from magnetic stimulation as being centrally rather than peripherally evoked. While, with the low stimulus intensity used, contraction of the target muscle alone did not sufficiently facilitate the cortically evoked response, this was the case when additional muscles, such as the tongue or the masseter, were contracted.

The central motor conduction time of $5.1 \mathrm{~ms}$ between cortex and the intratemporal nerve segment obtained by this technique (table 4) comprises an intracisternal and intratemporal peripheral segment accounting for a delay of some 0.4 to $0.7 \mathrm{~ms}(=10$ to $22 \mathrm{~mm}$ ) which must be considered when dealing with a pathology located here.

The method of exciting facial muscles by motor cortex stimulation was particularly interesting in the patients who had recovered from Bell's palsy. In these patients who failed to generate a response to magnetic facial nerve stimulation on the formerly affected side in spite of a normal function as judged from clinical examination, a normal response was obtained from cortical stimulation (figure 5). This clearly confirms that the inability to stimulate the peripheral nerve by the magnetic pulse is due to a hypo-excitable area in an otherwise normally conducting nerve.

We thank Professors Reulen and Seiler for permission to study their patients, Professor Ludin for inspiring discussions, Ms Sigron for technical assistance and Dr Koppi for valuable help during the experimental work. This study was supported by the Swiss National Science Foundation Numbers $3.852-1.86$ and 3.884 1.86 . 


\section{References}

1 Esslen E. The acute facial palsies. Berlin: Springer Verlag, 1977.

2 Mamoli B. Zur Prognosestellung peripherer Fazialisparesen unter besonderer Berücksichtingung der Elektroneurographie. Wiener klin Wochenschrift 1976;Suppl 53:1-28.

3 Murray NMF, Hess CW, Mills KR, Schriefer TN, Smith SJM. Proximal facial nerve conduction using magnetic stimulation. Electroenceph clin Neurophysiol 1987; 66:S71.

4 Schriefer TN, Mills KR, Murray NMF, Hess CW. Evaluation of proximal facial nerve conduction by transcranial magnetic stimulation. J Neurol Neurosurg Psychiatry 1988;51:60-6.

5 Barker AT, Jalinous R, Freeston IL. Non-invasive magnetic stimulation of the human motor cortex. Lancet 1985;i:1106-7.

6 Benecke R, Meyer BU, Schönle P, Conrad B. Transcranial magnetic stimulation of the human brain: responses in muscles supplied by cranial nerves. Exp Brain Res 1988;71:623-32.

7 Maccabee PJ, Amassian VE, Cracco RQ, Cracco JB, Anziska BJ. Intracranial stimulation of facial nerve in humans with the magnetic coil. Electroenceph clin Neurophysiol 1988;70:350-4.

8 Hess CW, Mills KR, Murray NMF. Magnetic stimulation of the human brain: facilitation of motor responses by voluntary contraction of ipsilateral and contralateral muscles with additional observations on an amputee. Neurosci Lett 1986;71:235-40.

9 Schmid UD, Sturzenegger M, Ludin HP, Seiler RW,
Reulen HJ. Orthodromic (intra/extracranial) neurography to monitor facial nerve function intra- $\mathbb{D}$ operatively. Neurosurgery 1988;22(5):945-50.

10 Hess CW, Mills KR, Murray NMF. Responses in small hand muscles from magnetic stimulation of the human brain. J Physiol (London) 1987;388:397-419.

11 Hess CW, Mills KR, Murray NMF, Schriefer TN. O Magnetic brain stimulation: central motor conduction $\lesssim$ studies in multiple sclerosis. Ann Neurol 1987;22: 744-52.

12 Merton PA, Morton HB. Stimulation of the cerebral cortex in the intact human subject. Nature 1980; $\overrightarrow{\bar{N}}$ 285:227.

13 Bickford RG, Dodge HW Jr, Uihlein A. Electrographic and behavioural effects related to depth stimulation in human patients. In: Ramey ER, O'Doherty DS, eds. New York: Harper, 1960:248-52.

14 Mills KR, Hess CW, Murray NMF. Magnetic scalp stimulation cannot be supramaximal. Electroenceph clin Neurophysiol 1987;66:S68.

15 Moeller AR, Janetta PJ. On the origin of synkinesis in hemifacial spasm: result of intracranial recording. $J \vec{\omega}$ Neurosurg 1984;61:569.

16 Kudo H, Nori S. Topography of the facial nerve in the human temporal bone. Acta Anatomica 1974;90(3): 467-80.

17 Ranck JB Jr. Specific impedance of rabbit cerebral cortex. Exp Neurol 1963;7:144-52.

18 Lang J. Klinische Anatomie des Kopfes. Berlin: Springe Verlag, 1981:386-9.

19 Agnew WF, McCreery DB. Consideration for safety the use of extracranial stimulation for motor evoke responses. Neurosurgery 1987;20(1):143-6. 